A LETTERS JOURNAL EXPLORING THE FRONTIERS OF PHYSICS

\title{
OFFPRINT
}

\section{Deformation of acoustically transparent fluid interfaces by the acoustic radiation pressure}

B. Issenmann, A. Nicolas, R. Wunenburger, S. Manneville and J.-P. DELVILLE

EPL, 83 (2008) 34002

Please visit the new website www.epljournal.org 


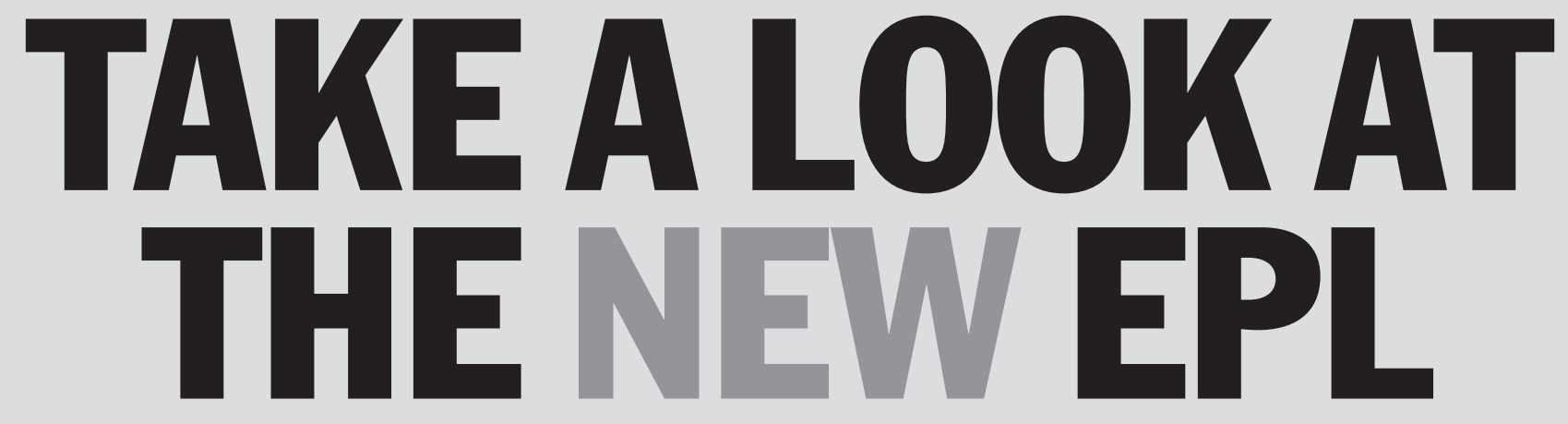

\section{Europhysics Letters (EPL) has a new online home at www.epljournal.org}

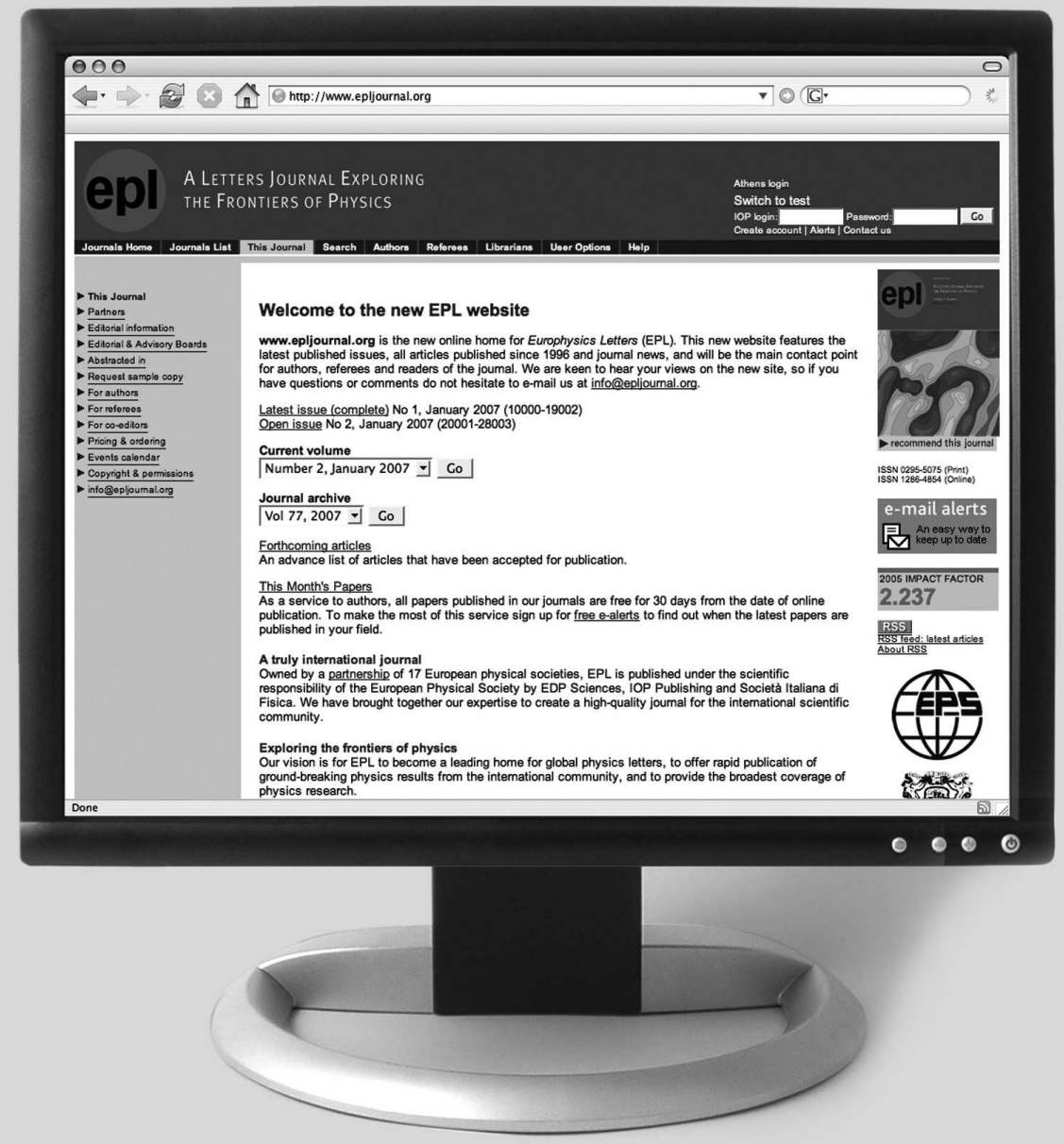

Take a look for the latest journal news and information on:

- reading the latest articles, free!

- receiving free e-mail alerts

- submitting your work to EPL 


\title{
Deformation of acoustically transparent fluid interfaces by the acoustic radiation pressure
}

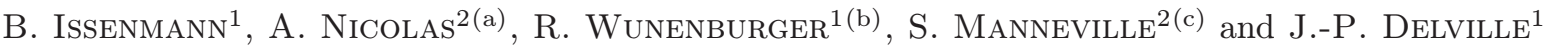 \\ ${ }^{1}$ Centre de Physique Moléculaire Optique et Hertzienne, UMR CNRS 5798, Université Bordeaux I \\ 351 cours de la Libération, 33405 Talence Cedex, France, EU \\ ${ }^{2}$ Centre de Recherche Paul Pascal, UPR CNRS 8641 - Avenue Schweitzer, 33600 Pessac, France, EU
}

received 26 March 2008; accepted in final form 13 June 2008

published online 18 July 2008

PACS 43.25.+y - Nonlinear acoustics

PACS 47.35.Pq - Capillary waves

PACS $47.20 . \mathrm{Ma}$ - Interfacial instabilities (e.g., Rayleigh-Taylor)

\begin{abstract}
We experimentally study the deformations of liquid-liquid interfaces induced by a high-intensity focused ultrasonic beam. We quantitatively verify that small-amplitude deformations of a transparent chloroform-water interface are well described by the theory of Langevin acoustic radiation pressure, in both static and dynamic regimes. The large-amplitude deformations depend on the direction of propagation of the beam and are qualitatively similar to those induced by electromagnetic radiation pressure.
\end{abstract}

Copyright (C) EPLA, 2008

Introduction. - Since the first theoretical description of the acoustic radiation pressure (RP) by Rayleigh in 1902 [1], several theoretical works have been devoted to its formulation in various configurations and using different approaches (for reviews, see $[2,3]$ ). Among the pioneers, Brillouin was the first to propose a tensorial formulation of the anisotropic radiation stress [4], whereas Langevin independently described the RP exerted by an acoustic beam of finite width on a perfectly reflecting obstacle irradiated at normal incidence [5]. The acoustic radiation stress is defined as the time-averaged force per unit surface area exerted by an acoustic wave on a given obstacle; it reduces to a normal force in some situations, including one-dimensional (1D) propagation (normal incidence). Its derivation fundamentally depends on the boundary conditions imposed to the acoustic propagation, as illustrated in the $1 \mathrm{D}$ case. The conservation of the velocity circulation in inviscid fluids implies that, to second order in $v / c,(v$ is the fluid velocity amplitude, $c$ the sound speed $)$, the time-averaged Lagrangian pressure $\left\langle P^{L}\right\rangle$, whose difference on both sides of the obstacle equals the RP in the $1 \mathrm{D}$ case, satisfies the equality $\left\langle P^{L}\right\rangle=\langle E\rangle+C$, where $\langle E\rangle$ is the time-averaged acoustic energy density and $C$ a

\footnotetext{
(a) Present address: Laboratoire des Technologies de la Microélectronique, UMR CNRS 5129 - 17 avenue des Martyrs, 38054 Grenoble cedex, France, EU.

(b) E-mail: $r$. wunenburger@cpmoh .u-bordeaux1.fr

(c) Present address: Ecole Normale Supérieure de Lyon, UMR CNRS 5672 - 46 allée d'Italie, 69364 Lyon cedex 07, France, EU.
}

constant [5]. In the case of an acoustic wave confined in a closed space with rigid boundaries (called Rayleigh's case), $C$ is determined by the constraint of fixed fluid volume in a subtle manner, implying that the Rayleigh RP depends not only on $\langle E\rangle$ but also on the nonlinearity of the fluid equation of state. In the case of an acoustic beam of finite width propagating in an extended fluid (called Langevin's case), which is more usual, $C$ simply equals the pressure of the fluid at rest outside the beam, and the Langevin RP depends only on $\langle E\rangle[2,3]$. For historical reasons, and also because the RP of focused beams acting on solid targets is now commonly used to calibrate high-intensity ultrasonic sources [6], most of works mainly consider perfectly reflecting or perfectly absorbing obstacles irradiated at normal incidence. The case of transparent or partially reflecting interfaces separating two fluids has been much less studied $[2,3,7,8]$, although it concerns several hydrodynamic phenomena induced by high-intensity acoustic waves observed since the 1920s $[9,10]$.

Paradoxically, despite the long-lasting theoretical controversies concerning both the Rayleigh RP, due to the unsuspectedly strong effect of the "acoustic straining", and the Langevin RP, due to methodological disagreements $[2,3]$, the acoustic RP has been the subject of very few experimental studies. The Rayleigh RP was carefully measured in gases by Mathiot [11], who checked the validity of Brillouin's theory on a perfectly reflecting solid target. The Langevin radiation stress was measured on perfectly absorbing and perfectly reflecting solid 


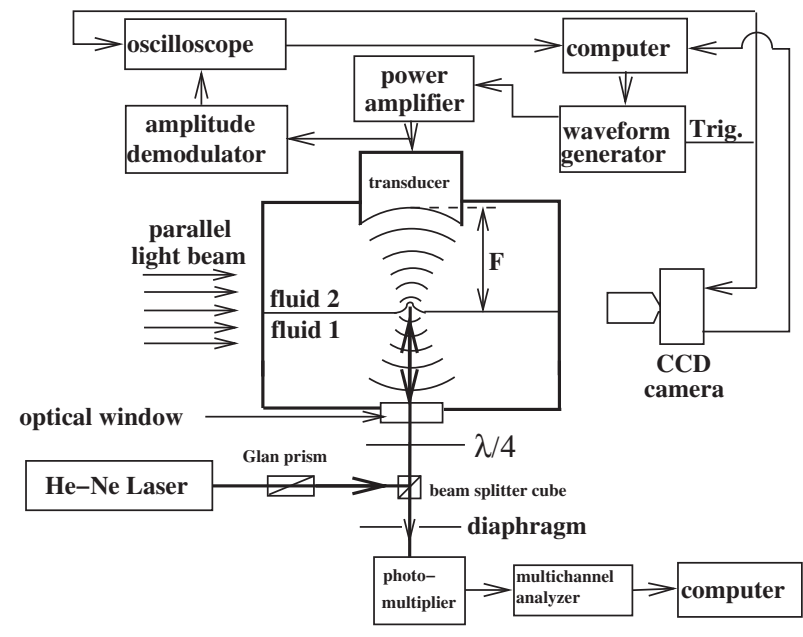

Fig. 1: Experimental setup. Its upper part was used for steadystate measurements, its lower part for dynamic measurements (see text).

targets irradiated at varying incidence by Herrey [12], who verified that the radiation stresses were actually anisotropic. Rooney experimentally confirmed the prediction that the Langevin RP acting on an absorbing solid target is independent of the acoustic nonlinear parameter of the fluid [13]. Hertz and Mende were the first to observe the deformations of transparent or partially reflecting liquid interfaces by the acoustic RP [10]. But no quantitative test of the prediction of the Langevin RP acting on a transparent or partially reflecting interface separating two extended media, on which refs. [2,3,7] and independently [8] agree, has been carried out so far. Nevertheless, this situation represents a crucial test of the theory since the acoustic beam crosses the interface and propagates in both media. More generally, the effect of a high-intensity acoustic wave on a liquid-liquid interface raises various other open questions, such as the exact shape of the deformation at steady state as a function of acoustic intensity. In this context, the goals of this paper are i) to experimentally check the validity of the expression of the Langevin RP acting on a transparent interface at normal incidence, and ii) to investigate the large-amplitude deformations of a liquid-liquid interface.

Experimental setup. - The experimental setup is sketched in fig. 1. An immersed spherical ultrasonic transducer vertically emits an acoustic beam focused on the interface between two immiscible fluids. The fluids are chosen so that the interface between them is acoustically transparent, i.e. their acoustic impedances are matched: $\rho_{1} c_{1} \simeq \rho_{2} c_{2}, \rho_{i}$ being the density of fluid $i$, and $c_{i}$ its sound velocity. In most of this study, experiments are performed on the interface between water and chloroform (Acros Organics, stabilized with $1 \%$ ethanol). The interface pressure reflection coefficient at normal incidence is $r=0.7 \%$. We shall also briefly present some results obtained on the interface between water and a silicon oil (DC704 from
Table 1: Physical properties of the (chloroform $+1 \%$ ethanol)water and DC704 oil-water interfaces at temperature $25^{\circ} \mathrm{C}$. The density and viscosity of DC704 oil are those indicated by the manufacturer Dow Corning.

\begin{tabular}{ccc}
\hline Fluid 1 & Chloroform & DC704 oil \\
Fluid 2 & Water & Water \\
Density $\rho_{1}\left(\mathrm{~kg} / \mathrm{m}^{3}\right)$ & $1490[14]$ & 1070 \\
$\rho_{2}\left(\mathrm{~kg} / \mathrm{m}^{3}\right)$ & $997[14]$ & 997 \\
Sound speed $c_{1}(\mathrm{~m} / \mathrm{s})$ & $987[14]$ & $1411 \pm 1$ \\
$c_{2}(\mathrm{~m} / \mathrm{s})$ & $1497[14]$ & 1497 \\
Viscosity $\eta_{1}(\mathrm{~Pa} \mathrm{~s})$ & $5.4 \cdot 10^{-4}[14]$ & $4.2 \cdot 10^{-2}$ \\
$\eta_{2}(\mathrm{~Pa} \mathrm{~s})$ & $8.9 \cdot 10^{-4}[14]$ & $8.9 \cdot 10^{-4}$ \\
$\sigma(\mathrm{N} / \mathrm{m})$ & $(20 \pm 1) \cdot 10^{-3}$ & $(18 \pm 1) \cdot 10^{-3}$ \\
\hline
\end{tabular}

Dow Corning) for which $r=0.6 \%$. Because the experimental test of the model of RP crucially depends on the interfacial tension $\sigma$, we took care in measuring it for the various interfaces [15]. The physical properties of the liquids under study are gathered in table 1 .

A glass tank filled with chloroform and pure degassed water is closed with PTFE caps at both ends. The transducer was previously sealed at the bottom or at the top cap, depending on the experiment to be performed. We preferably placed it on the water side of the tank and covered it by a thin layer of spray-PTFE protecting the epoxy impedance matching layer from the chloroform. The volumes of the two fluids were adjusted so that the interface lies in the focal plane of the transducer to within $1 \mathrm{~mm}$.

The transducer is a spherical Imasonic ultrasonic transducer (focal length $F=38 \mathrm{~mm}$, aperture $N A=1$, $f=2.25 \mathrm{MHz}$ central frequency, $600 \mathrm{kHz}$ bandwidth, focal zone length $l=4 \mathrm{~mm}, 5 \mathrm{~s}$ maximal sonication time). It is fed by an Amplifier Research 75A250 power amplifier driven by a PC-controlled Agilent 33250A waveform generator. The instantaneous amplitude of the amplifier output signal is extracted using a home-made amplitude demodulator, and sent to a digital Lecroy 9361 oscilloscope. Independent pressure measurements in a large water tank showed that the amplitude $P_{0}(r)$ of the pressure field $P(r, t)$ in the focal plane of the transducer is well described by the usual expression for spherical transducers $\quad P_{0}(r)=P \phi(r)=2 P\left|J_{1}(\pi r / \lambda) /(\pi r / \lambda)\right| \quad[16]$. $J_{1}$ is the first-order Bessel function, $r$ is the radius in cylindrical coordinates, and $\lambda=660 \mu \mathrm{m}$ is the acoustic wavelength in water at central frequency. Typical pressure amplitudes at focus lie between $P=0.1$ and $4 \mathrm{MPa}$. The liquid interface is illuminated at grazing incidence by a parallel white light beam, and pictures of the interface are captured using a high-speed Redlake Imaging MotionMeter CCD camera triggered by the waveform generator. For continuous and moderate acoustic intensities, the observed interface deformations are stationary, bell-shaped, and of diameter at half-height comparable to the diameter at half-maximum in energy of the acoustic beam, $650 \mu \mathrm{m}$ (fig. 2). 


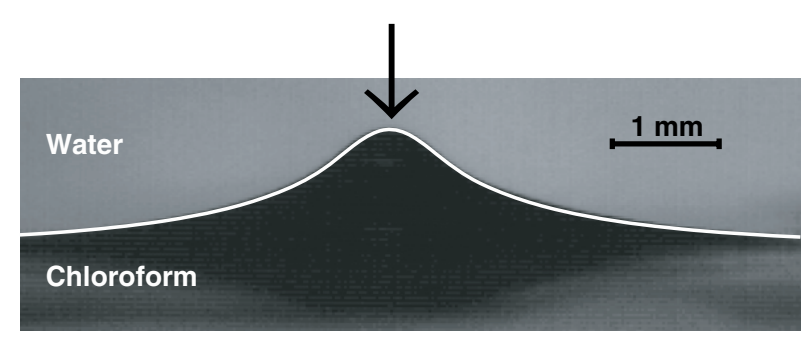

Fig. 2: Photograph of the observed steady-state deformation of the chloroform-water interface. The white line is the theoretical profile predicted by eq. (2). The incident acoustic wave comes from water (black arrow). The measured acoustic pressure amplitude at focus is $1.17 \mathrm{MPa}$ and the frequency is $2.25 \mathrm{MHz}$. The acoustic pressure amplitude used to compute the theoretical profile of the deformation is $1.06 \mathrm{MPa}$, in accordance with $14 \%$ uncertainty on the pressure measurement (see the "steady-state measurements" section).

Steady-state interface deformations induced by radiation pressure. - As explained in the introduction, assigning the index 1 (respectively, 2) to the bottom (respectively, the top) fluid, the Langevin RP $\Pi$ applied upwards on an interface separating two media by a beam of finite width impinging the interface at normal incidence equals the difference of time-averaged acoustic Lagrangian pressures $\left\langle P^{L}\right\rangle$ on both sides of the interface $\Pi(r)=\left\langle P_{1}^{L}(r)\right\rangle-\left\langle P_{2}^{L}(r)\right\rangle$ and also writes $\Pi(r)=$ $\left\langle E_{1}(r)\right\rangle-\left\langle E_{2}(r)\right\rangle$. At a transparent interface, $E_{i}$ is simply the energy density of the plane progressive wave propagating in fluid $i$ and then $\Pi(r)=\left(\chi_{S 1}-\chi_{S 2}\right) P_{0}^{2}(r) / 2$, where $\chi_{S i}=1 / \rho_{i} c_{i}^{2}$ is the isentropic compressibility of fluid $i$. In the general case, the interface might be not transparent and the radiation pressure writes $\Pi(r)=A P_{0}^{2}(r)$, where $A=\varepsilon \frac{1}{\rho_{i} c_{i}^{2}} \frac{\rho_{i}^{2} c_{i}^{2}+\rho_{j}^{2} c_{j}^{2}-2 \rho_{i} \rho_{j} c_{i}^{2}}{\left(\rho_{i} c_{i}+\rho_{j} c_{j}\right)^{2}}$, where $\varepsilon=1, i=1, j=2$ (respectively, $\varepsilon=-1, i=2, j=1$ ) if the incident beam propagates upward (respectively, downward) $[2,3,7,8]$.

In order to check the validity of this prediction, we direct moderate acoustic intensity perpendicularly to an acoustically transparent liquid-liquid interface in order to generate axisymmetric interface deformations of small amplitude (i.e. of slope $\left|h^{\prime}(r)\right|$ such that $\left|h^{\prime}(r)\right| \ll 1, h(r)$ being the height profile of the deformation) and assume the incident acoustic beam to always impinge the interface at normal incidence. The shape $h(r)$ of the steady-state deformation results from the competition between the RP, that tends to deform the interface, and the gravity and the Laplace pressure, that tend to flatten it

$$
\left(\rho_{1}-\rho_{2}\right) g h(r)-\sigma \kappa(r)=\Pi(r),
$$

$g$ being the acceleration of gravity and $\kappa(r)$ the curvature of the interface at a distance $r$ from the axis of the acoustic beam. For small-amplitude deformations, $\kappa \approx \frac{1}{r} \frac{\mathrm{d}}{\mathrm{d} r}\left(r \frac{\mathrm{d} h}{\mathrm{~d} r}\right)$. The solution of eq. (1) writes $[17,18]$

$$
h(r)=4 A P^{2} \int_{0}^{2 \pi / \lambda} \frac{\tilde{\pi}(k)}{\left(\rho_{1}-\rho_{2}\right) g+\sigma k^{2}} J_{0}(k r) k \mathrm{~d} k,
$$

where $\tilde{\pi}(k)$ is the Hankel transform of $\phi^{2}(r)$ :

$$
\left\{\begin{array}{l}
\tilde{\pi}(k) \stackrel{k<2 \pi / \lambda}{=} \frac{2 \lambda^{2}}{\pi^{2}}\left(1-\frac{\lambda k}{\pi^{2}} \sqrt{1-\frac{k^{2} \lambda^{2}}{4 \pi^{2}}}-\frac{2}{\pi} \arcsin \left(\frac{\lambda k}{2 \pi}\right)\right), \\
\tilde{\pi}(k) \stackrel{k \geqslant 2 \pi / \lambda}{=} 0 .
\end{array}\right.
$$

Note that $A \simeq \frac{1}{2}\left(\chi_{S 1}-\chi_{S 2}\right)$ within $4 \%$ in the case of the water-chloroform interface. According to eq. (2), the height of the deformation tip $h=h(r=0)$ is proportional to the square of the acoustic pressure amplitude $P$ at the transducer focus (despite the nonlinearity of eq. (1), this proportionality holds to within $1.7 \%$ in the pressure range used for the experimental check of the prediction) and its sign does not depend on the direction of propagation of the incident beam: whatever the fluid the incident beam comes from, the interface always deforms in the same manner towards the less compressible fluid, as already observed by Hertz and Mende [10] (up-down invariance). In the following, we experimentally check the validity of these theoretical predictions by comparing the steadystate interface deformations shapes and heights to eq. (2). To our knowledge, the present experiments constitute the first attempt to test these prediction quantitatively.

Steady-state measurements. - The acoustic pressure amplitude $P$ at the focus of the transducer is proportional to the amplitude $V$ of the sine voltage of frequency $2.25 \mathrm{MHz}$ feeding the transducer. We define $P$ as the amplitude of the fundamental mode of the pressure at the focus. In fact, in the investigated pressure ranges, the first harmonic amplitude is less than $2 \%$ of the fundamental one and then can be neglected. The linear relationship between $V$ and $P$ was experimentally determined using a Precision Acoustics needle hydrophone of sensitive element diameter $40 \mu \mathrm{m}$ and sensitivity $3.9 \mathrm{mV} / \mathrm{MPa}$, with a specified $14 \%$ uncertainty. During an experiment, $P^{2} \propto V^{2}$ is ramped linearly up and down for $5 \mathrm{~s}$ in order to verify that no hysteresis phenomenon appears, contrary to the case of a totally reflecting interface $[16] ; V=0$ the rest of the time. The characteristic response time of the deformation being of order $60 \mathrm{~ms}$ (see fig. 5), the deformation is assumed to evolve quasistatically during the ramps so that its instantaneous axisymmetric shape should be described by eq. (2). It can be checked in fig. 2 that the predicted shape is in excellent agreement with the observed deformation. The signal out from the amplitude demodulator, which is recorded by the oscilloscope, is proportional to $V$ and thus allows us to determine the instantaneous pressure amplitude $P$ of the incident acoustic wave. This signal as well as the pictures taken by the camera are recorded simultaneously. This allows us to experimentally determine the variation of $h$ as a function of $P^{2}$. We checked that the $h\left(P^{2}\right)$ variations were independent of the initial distance $d$ between the flat interface and the focus for $d<1 \mathrm{~mm}$ and $h<1 \mathrm{~mm}$, insuring that the entire deformation remains within the focal zone. The comparison with the prediction of eq. (2) for the water-chloroform interface 


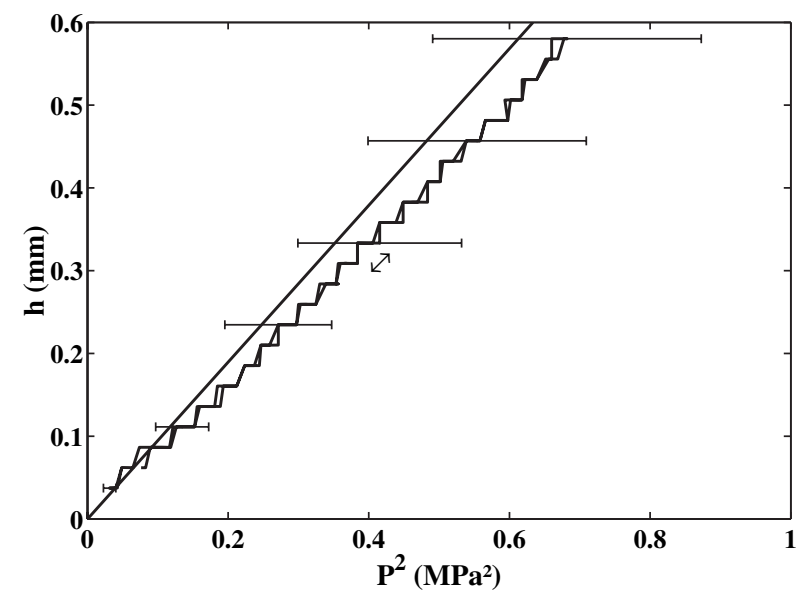

Fig. 3: Height of the deformation as a function of the squared amplitude of the incident wave for an up and down linear ramp in $P^{2}$ (indicated by the double arrow). The straight line is $h(r=0)$ inferred from eq. (2) for the water-chloroform interface. The experimental curve is discretised due to the discretisation of the amplitude demodulator and to the pixelation of the camera. As indicated by the horizontal error bars, the experimental pressure amplitude is measured within a $14 \%$ uncertainty due to the needle hydrophone calibration. The acoustic wave was emitted from water.

is shown in fig. 3. Taking into account the uncertainty on $P$ due to the calibration, the experimental curve $h\left(P^{2}\right)$ is in quantitative agreement with eq. (2). Note that this prediction involves no free parameter. Finally, we experimentally confirmed the up-down invariance. Before definitively concluding about the validity of the Langevin theory for the RP, we have to consider other possible forces acting on the fluid interface.

Role of acoustic streaming. - When an acoustic wave propagates through a dissipative medium, a streaming flow sets in within this medium [19]. Such acoustic streaming develops in both fluids used in our experiments and may exert normal surface stresses on the interface that might contribute to part of the interface deformation. The order of magnitude of the normal viscous stresses exerted on the interface by acoustic streaming in the fluid $i$ is expected to be $\Pi_{i}^{\prime} \sim \eta_{i} \frac{\partial u_{s i}}{\partial x_{i}} \sim \eta_{i} \frac{u_{s i}}{w}, \eta_{i}$ being the dynamic viscosity of fluid $i, u_{s i}$ the typical velocity of acoustic streaming in fluid $i$ and $w$ the characteristic length scale of variation of $u_{s i}$, which is of the order of the beam width, here $\lambda$. In a bulk fluid and for $R e_{s t} \ll 1$, where $R e_{s t}$ is the Reynolds number associated to the streaming flow, the streaming velocity $u_{s}$ at the focus of an acoustic beam of focal zone length $l$ is [19]

$$
u_{s}=\frac{\alpha w^{2}}{4 \eta \rho c^{2}} P^{2} \ln \left(\frac{\sqrt{4 w^{2}+l^{2}}+l}{\sqrt{4 w^{2}+l^{2}}-l}\right) .
$$

$\alpha$ is the attenuation coefficient of the acoustic wave and writes for liquids $[20] \alpha \simeq \frac{2 \pi^{2} f^{2}}{\rho c^{3}}\left(\frac{4}{3} \eta+\eta^{\prime}\right) . \eta^{\prime}$ is the fluid bulk viscosity. Here we apply eq. (4) to the case
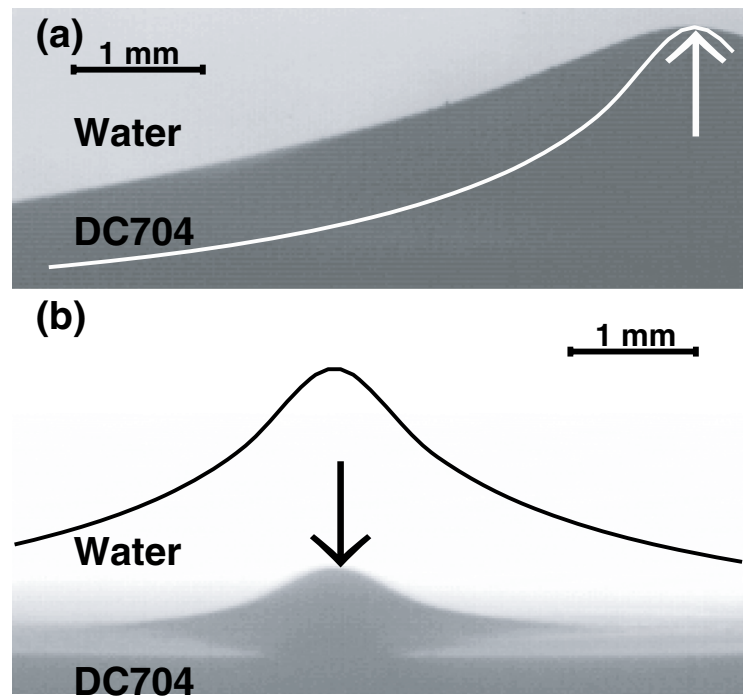

Fig. 4: Photographs of the observed deformation of the DC704water interface. (a) The acoustic wave comes from DC704 (white arrow). The white line is the theoretical profile predicted by eq. (2). The acoustic pressure amplitude is $3.6 \mathrm{MPa}$ and the frequency is $2.25 \mathrm{MHz}$. The origin in heights was arbitrarily chosen so that the top of the deformation coincides with the top of the theoretical curve. (b) The acoustic wave comes from water (black arrow). The black line is the theoretical profile predicted by eq. (2). The acoustic pressure amplitude is $3.5 \mathrm{MPa}$ and the frequency is $2.25 \mathrm{MHz}$.

of a focused beam impinging a liquid-liquid interface located at its focus in order to get a rough estimate for $\Pi_{i}^{\prime}$. The values of $\eta^{\prime}$ for chloroform and water can be deduced from the values of $n=\eta$ and $n^{\prime}=\eta^{\prime}-\frac{2}{3} \eta$ given in ref. [21]: $\eta_{\text {water }}^{\prime} \approx 3 \eta_{\text {water }}$ and $\eta_{\text {chloroform }}^{\prime} \approx 25 \eta_{\text {chloroform }}$. According to ref. [21], an order of magnitude of $\eta^{\prime} / \eta$ for liquids is 10, a ratio which will be applied to the DC704 silicon oil. We expect the influence of acoustic streaming on the interface to be negligible compared to the RP if the ratio $r_{i}=\Pi / \Pi_{i}^{\prime}$ is much larger than 1 for both fluids. Note that, at fixed frequency, $r_{i}$ does not depend on the amplitude $P$ of the acoustic wave, but only on the fluid properties.

In the case of a chloroform-water interface, $r_{1}=300$ and $r_{2}=4000$, so that normal surface stresses exerted on this interface by the streaming flow should be negligible in comparison to the acoustic RP. To further check the possibility that the effect of acoustic streaming induced interface stresses may in some cases become important, we performed the same experiments on an acoustically transparent silicon oil DC704-water interface $\left(\rho_{1} c_{1} \approx \rho_{2} c_{2}\right)$, furthermore presenting a weak compressibility contrast, $\left|\chi_{S 1}-\chi_{S 2}\right| \ll \chi_{S 1}$, i.e., on which the RP is expected to be intrinsically weak (see eq. (2)). As a matter of fact, $r_{1}=3$ and $r_{2}=500$ for this interface, so that a noticeable mechanical effect of acoustic streaming on this interface is expected. This is confirmed by figs. $4 \mathrm{a}$ and $\mathrm{b}$ where pictures of DC704-water interface deformations 


$$
\alpha(t)=P^{2} \frac{A\left(\rho_{1}+\rho_{2}\right)}{\left(\eta_{1}+\eta_{2}\right)^{2}} \int_{0}^{2 \pi / \lambda} \tilde{\pi}(k) \beta(k) \frac{\omega^{2}(k)}{\Omega(k)} \frac{\Omega(k)+\exp (-2 \omega(k) t)(-2 \omega(k) \sin (\Omega(k) t)-\Omega(k) \cos (\Omega(k) t))}{4 \omega^{2}(k)+\Omega^{2}(k)} \mathrm{d} k,
$$

obtained with both upward and downward propagating acoustic beams of comparable intensities are shown. Here $h$ strongly depends on the direction of propagation, contrary to the prediction, and both deformation profiles computed using eq. (2) do not fit the observed interface deformations.

Interfacial dynamics. - In order to definitely discard possible mechanical effects other than the RP, we study the dynamics of the water-chloroform interface deformations and compare it to a model of interfacial dynamics assuming the acoustic RP as the only surface stress acting on the interface. This model is adapted from Ostrovskaia's theory of free-surface deformation by optical RP [22]. In this theory based on the linearized Navier-Stokes equation, we consider the small-amplitude deformation of a two-fluid interface by the RP of a focused acoustic wave impinging the interface at normal incidence. The RP is turned on at $t=0$, then kept constant. As the characteristic time of the deformation growth is much larger than the period of the acoustic wave, the flow is considered as incompressible. The time evolution $h(t)$ of the height of the deformation and the curvature $\kappa(t)$ of the deformation tip are given by

$$
\text { see eq. (5) above }
$$

where $\beta(k)=\frac{1}{k^{2}}$ when $\alpha(t)=h(t)$ and $\beta(k)=-1$ when $\alpha(t)=\kappa(t)$.

$1 / \omega(k)=\left(\rho_{1}+\rho_{2}\right) /\left(\left(\eta_{1}+\eta_{2}\right) k^{2}\right) \quad$ is the characteristic time of viscous diffusion of momentum over the characteristic length scale $2 \pi / k$, and $1 / \Omega(k)=1 / \sqrt{\left(\rho_{1}-\rho_{2}\right) g k /\left(\rho_{1}+\rho_{2}\right)+\sigma k^{3} /\left(\rho_{1}+\rho_{2}\right)} \quad$ is the characteristic time scale of the inertial dynamics of an interface deformation of characteristic length scale $2 \pi / k$. To obtain eq. (5), we assumed the interfacial dynamics to be inertial, i.e. $\Omega(k) / \omega(k) \gg 1$ for any $k<2 \pi / \lambda$, a valid assumption in the case of the water-chloroform interface, since $\Omega(k) / \omega(k)$ is a decreasing function of $k$ and $\Omega(2 \pi / \lambda) / \omega(2 \pi / \lambda)=50$.

Acoustic streaming being associated to the viscous diffusion of vorticity over the acoustic wavelength $\lambda[23]$, the characteristic time scale $\tau_{v}$ of setting up of the acoustic streaming writes $\tau_{v}=\frac{\rho}{\eta k_{a c}^{2}}$, where $k_{a c}=2 \pi / \lambda$, and is equal to $13 \mathrm{~ms}$ in water and in chloroform. So, if acoustic streaming had a noticeable effect on the interface deformation, this effect would result in a departure of the measured $h(t)$ from its predicted behavior eq. (5) from $t \simeq \tau_{v}$. The quantitative agreement between the measured dynamics of the deformation height and its predicted behavior given by eq. (5) shown in fig. 5 leads to conclude that no effect of the acoustic streaming on the waterchloroform interface deformation height is detectable.

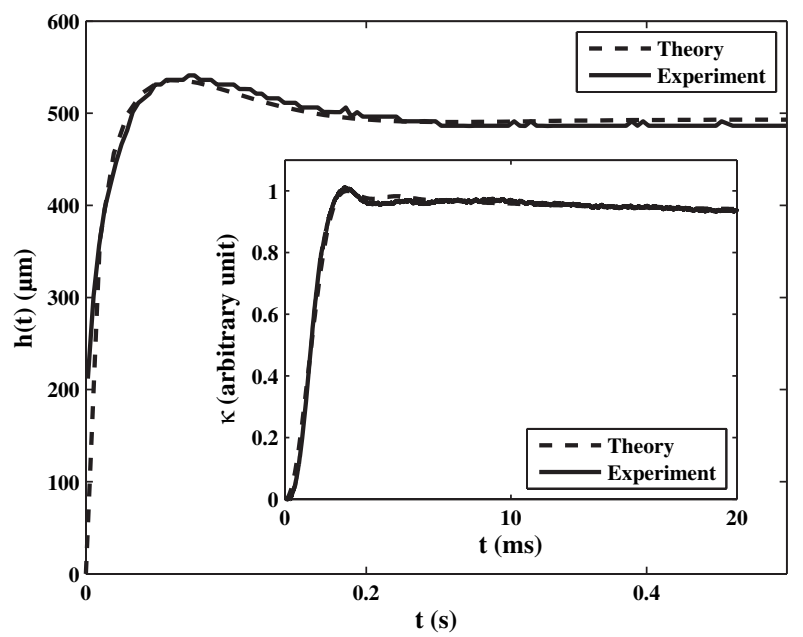

Fig. 5: Dynamic evolution of the height of the deformation. The acoustic frequency is $f=2.25 \mathrm{MHz}$ and the pressure amplitude is $P=0.77 \mathrm{MPa}$. The acoustic wave is emitted from water. The images are captured at $250 \mathrm{fps}$. Inset: dynamic evolution of the curvature of the deformation. The acoustic frequency is $f=2.25 \mathrm{MHz}$ and the pressure amplitude is $P=0.06 \mathrm{MPa}$. The acoustic wave is emitted from water.

Since the curvature of the deformation tip $\kappa(t)=\frac{1}{r} \frac{\partial}{\partial r}\left(r \frac{\partial h}{\partial r}\right)(0, t)$ undergoes faster dynamics than $h(t)$, we also sought for a possible manifestation of the acoustic streaming on the $\kappa(t)$ signal. To measure $\kappa(t)$ accurately, we used the focusing of a laser beam by the deformation tip acting as a partially reflecting convergent mirror. The experimental setup is shown in the lower part of fig. 1. A circularly polarized $17 \mathrm{~mW}$ He-Ne laser is first linearly polarized by a Glan prism. A beam splitter cube (BSC) directs the beam towards the interface, while a lens association (not shown in fig. 1) locates its waist on the interface. It is partially reflected by the interface. A $\lambda / 4$ plate allows to separate the reflected from the incident beam in the BSC. The reflected and focused beam is filtered through an iris diaphragm and its power is measured using a photomultiplier and a multi-channel analyzer. The interface acts as a spherical mirror of focal length $F=1 / \kappa$. We use sufficiently small acoustic intensities so that $F$ remains always much larger than the distance between the interface and the diaphragm. In these conditions, the variation of the light power $\mathcal{P}$ passing through the diaphragm is proportional to $\kappa[18]$. The quantitative agreement between the $\kappa(t)$ signal deduced from $\mathcal{P}(t)$ and its predicted behavior given by eq. (5) shown in the inset of fig. 5 confirms that even at short time scales no effect of the acoustic streaming on the water-chloroform interface is detectable. 


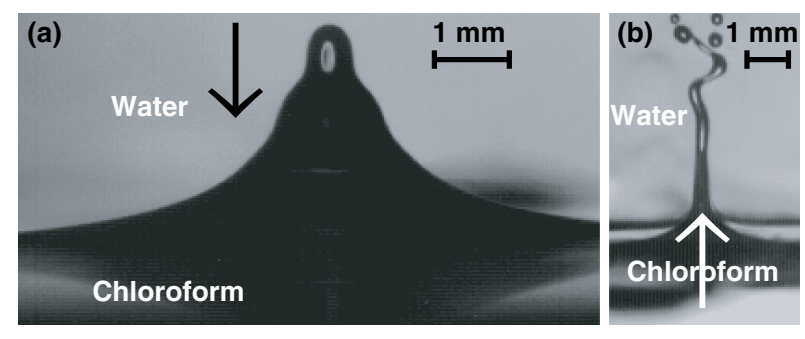

Fig. 6: (a) Tether-like shape observed at the interface between water and chloroform. The acoustic amplitude was $2.0 \mathrm{MPa}$ and the frequency was $2.25 \mathrm{MHz}$. The incident acoustic beam came from the top fluid (arrow). (b) Jet observed at the interface between water and chloroform. The acoustic amplitude was 1.3 MPa and the frequency was $2.25 \mathrm{MHz}$. The incident acoustic beam came from the chloroform (arrow). The jet is unsteady, swirls and emits droplets.

Consequently, the quantitative agreement between experiment and theory shown in figs. 2 and 3 together with the demonstration given by fig. 5 that acoustic streaming has no detectable effect on both the dynamics of the interface and its steady-state shape prove the validity of the theory of the Langevin $\mathrm{RP}$ acting on a transparent fluid interface.

Large-amplitude deformations. - In all the experiments presented so far, the acoustic amplitude was small enough to obtain bell-shaped deformations like the one shown in fig. 2. When a higher acoustic power is used, interface deformations exhibit two kinds of shapes, called hereafter "tethers" (fig. 6a) and "jets" (fig. 6b).

Very similar shapes have already been observed when focusing a laser beam on the interface between two immiscible liquids $[17,24]$. Like in the optical case, jets are observed beyond a power threshold in the configuration where the deformation grows in the direction of acoustic propagation, and tethers are observed in the configuration where the deformation grows in the opposite direction. Those shapes, which violate the up-down shape invariance predicted for small-amplitude deformations at normal incidence, are still not explained at the present time and will deserve future attention.

Conclusion. - The static and dynamic experiments presented in this article demonstrate the validity of the theory of the Langevin radiation pressure of an acoustic wave impinging a transparent fluid interface at normal incidence, in a case where the acoustic streaming was proven to have no significant influence on the shape of the deformation. Using more intense acoustic waves, we observed large-amplitude interface deformations violating the up-down shape invariance predicted for small-amplitude deformations and normal incidence. Since such shapes are observed when strong acoustic or optical radiation pressure acts on liquid-liquid interfaces, it is expected that they can be explained by a mechanism independent of the nature of the exciting wave.

$$
* * *
$$

The authors thank O. MisèRE for preliminary experiments. This work was supported by Centre National de la Recherche Scientifique, Université Bordeaux 1, Conseil Régional d'Aquitaine under Contract 20040205003A and ACI Jeunes Chercheurs No. 2046.

\section{REFERENCES}

[1] Lord Rayleigh, Philos. Mag., 3 (1902) 338.

[2] Chu B. and Apfel R. E., J. Acoust. Soc. Am., 72 (1982) 1673.

[3] Lee C. P. and Wang T. G., J. Acoust. Soc. Am., 94 (1993) 1099.

[4] Brillouin L., Ann. Phys. (Paris), 4 (1925) 528.

[5] Biquard P., Rev. Acoust., 1 (1932) 93.

[6] BeIssner K., in Ultrasonic Exposimetry, edited by ZISKIN M. and LEwIN P. (CRC) 1992, pp. 127-142.

[7] Borgnis F. E., Rev. Mod. Phys., 25 (1953) 653.

[8] Landau L. and Lifschitz E., Fluid Mechanics, 2nd edition (Butterworth-Heinemann) 1987.

[9] Wood R. W. and Loomis A. L., Philos. Mag., 4 (1927) 417.

[10] Hertz G. and Mende H., Z. Phys., 114 (1939) 354.

[11] Матнiot M., Ann. Phys. (Paris), 1 (1966) 235.

[12] Herrey E. M. J., J. Acoust. Soc. Am., 27 (1955) 891.

13] Rooney J. A., J. Acoust. Soc. Am., 54 (1973) 429.

[14] Weast Robert C., Handbook of Chemistry and Physics, 69th edition (CRC Press, Cleveland) 1988-1989.

[15] Our measure for the water-chloroform interface differs from that reported in DEMOND A. H. and LindNER A. S., Environ. Sci. Technol., 27 (1993) 2318, because the chloroform we used was stabilized with $1 \%$ ethanol.

[16] Issenmann B., Wunenburger R., Manneville S. and Delville J.-P., Phys. Rev. Lett., 97 (2006) 074502.

[17] Wunenburger R., Casner A. and Delville J.-P., Phys. Rev. E, 73 (2006) 036314, 036315.

[18] Issenmann B., Déformations d'interfaces fluides par la pression de radiation acoustique, $\mathrm{PhD}$ Thesis (Université, Bordeaux I, Bordeaux) (2007).

[19] Nyborg W. L., in Nonlinear Acoustics, edited by Blackstock D. T. and Hamilton M. F. (Academic Press) 1998, pp. 214-216.

[20] Kinsler L. E., Frey A. R., Coppens A. B. and SAnders J. V., Fundamentals of Acoustics (John Wiley and Sons) 2000, p. 213.

[21] Liebermann L. N., Phys. Rev., 75 (1949) 1415.

[22] Ostrovskaia G. V., Sov. Phys. Tech. Phys., 33 (1988) 465.

[23] Eckart C., Phys. Rev., 73 (1948) 68.

[24] Casner A., Delville J.-P. and Brevik I., J. Opt. Soc. Am. B, 20 (2003) 2355. 